\title{
Quantum metric and wavepackets at exceptional points in non-Hermitian systems
}

\author{
D. D. Solnyshkov, ${ }^{1,2}$ C. Leblanc,${ }^{1}$ L. Bessonart,${ }^{1}$ A. Nalitov, ${ }^{1,3}$ Jiahuan Ren, ${ }^{4,5}$ Qing Liao, ${ }^{4}$ F. Li, ${ }^{6}$ and G. Malpuech ${ }^{1}$ \\ ${ }^{1}$ Institut Pascal, PHOTON-N2, Université Clermont Auvergne, \\ CNRS, SIGMA Clermont, F-63000 Clermont-Ferrand, France. \\ ${ }^{2}$ Institut Universitaire de France (IUF), F-75231 Paris, France \\ ${ }^{3}$ Faculty of Science and Engineering, University of Wolverhampton, Wulfruna St, Wolverhampton WV1 $1 L Y$, UK \\ ${ }^{4}$ Beijing Key Laboratory for Optical Materials and Photonic Devices, Department of Chemistry, \\ Capital Normal University, Beijing 100048, People's Republic of China \\ ${ }^{5}$ Tianjin Key Laboratory of Molecular Optoelectronic Sciences, \\ Department of Chemistry, School of Sciences, Tianjin University, \\ Collaborative Innovation Center of Chemical Science and Engineering, Tianjin 300072, People's Republic of China \\ ${ }^{6}$ Key Laboratory for Physical Electronics and Devices of the Ministry \\ of Education 85 Shaanxi Key Lab of Information Photonic Technique, \\ School of Electronic and Information Engineering, \\ Xi'an Jiaotong University, Xi'an 710049, China
}

\begin{abstract}
The usual concepts of topological physics, such as the Berry curvature, are not always relevant for non-Hermitian systems. We show that another object, the quantum metric, which often plays a secondary role in Hermitian systems, becomes a crucial quantity near exceptional points in nonHermitian systems, where it diverges in a way that fully controls the description of wavepacket trajectories. The quantum metric behaviour is responsible for a constant acceleration with a fixed direction, and for a non-vanishing constant velocity with a controllable direction. Both contributions are independent of the wavepacket size.
\end{abstract}

\section{INTRODUCTION}

Non-Hermitian systems in quantum mechanics ${ }^{1}$ and optics $^{2}$ expand the spectrum of possibilities much beyond that of the Hermitian ones ${ }^{3,4}$. A topic of very strong interest is offered by the merging of the so-called topological and non-Hermitian physics. For weak nonHermiticity, when the energy bands remain separate in the whole space (e.g. Brillouin zone), it is still possible to use the Chern numbers and the associated bulk-boundary correspondence $^{5}$. On the contrary, if the non-Hermiticity is strong and the parameter space contains an exceptional point where the bands merge, the topological invariants of Hermitian systems are not relevant any more: it is not the Chern number, but the winding number of a complex effective field (and the associated complex eigenvalues), which determines the topology in the non-Hermitian case $^{6}$. Partially because of this, the quantum geometry of the eigenstates has not been studied extensively in such strongly non-Hermitian systems ${ }^{7-9}$. This geometry is described by the quantum geometric tensor ${ }^{10}$ (QGT), which includes the Berry curvature (the cornerstone of Hermitian topological physics) and the quantum metric. Even in Hermitian systems, the whole QGT has been measured experimentally relatively recently ${ }^{11,12}$ and the quantum metric often plays a secondary role with respect to the ubiquitous Berry curvature. It is so far used in the calculations of quantum phase transitions ${ }^{13}$, electronic orbital magnetic susceptibility ${ }^{14,15}$, excitonic levels ${ }^{16}$, and superfluidity in flat bands ${ }^{17,18}$. The anomalous Hall effect, corresponding to a lateral shift of a wavepacket (WP) evolving adiabatically within a single dispersion branch, involves both components of the QGT, but the dominant role is played by the Berry curvature, while the quan- tum metric appears as a correction ${ }^{14,19}$. The situation is different in vicinity of the exceptional points in nonHermitian systems. Such points arise where the Hermitian and non-Hermitian parts of the Hamiltonian exactly compensate each other. The exceptional points can be encircled either statically (in so-called "stroboscopic" experiments) or dynamically, by changing the Hamiltonian parameters over time. In the first case, one has the access to all branches of the complex energy dispersion, and the topological winding numbers ${ }^{5,6}$ can be studied ${ }^{20}$. In the second case, the non-Hermitian properties of the system bring chirality into the dynamics of the system, making the exponentially decaying eigenstates completely inaccessible adiabatically ${ }^{21,22}$. In particular, it is actually impossible to encircle the exceptional point twice in any direction $^{23}$. While from the Hermitian point of view, this may seem as a flaw, one can actually take advantage of this feature to construct chiral optical transmitters ${ }^{24,25}$. The non-Hermitian systems thus require switching to a new way of thinking. Because of this, very recent studies started to focus on the divergence of the quantum $\operatorname{metric}^{9,26}$.

Many different experimental implementations of exceptional points are currently studied in photonics ${ }^{2}$. They include coupled waveguides and resonators, as well as various lattices ${ }^{27,28}$, with foreseen applications such as enhanced sensors ${ }^{29}$ and quantum information ${ }^{30}$. Microcavities ${ }^{31}$, with their widely tunable properties, represent a particularly versatile platform for nonHermitian physics ${ }^{32,33}$. The singular optical axes, also called Voigt points ${ }^{34}$, represent a particularly interesting configuration, fascinating scientists for more than a century ${ }^{35}$. They appear in optical systems combining spin-orbit coupling and polarization-dependent 
absorption $^{36,37}$. Recently, the square root topology of such points has been demonstrated in a ZnO-based microcavity ${ }^{38,39}$. Contrary to other non-Hermitian systems, many of which are described by a synthetic parameter space (e.g. coupling constant, detuning, and gain/loss for coupled resonators), these points occur in the reciprocal space describing the direction of the propagation of a beam. This allows to study effects linked with the WP dynamics in real and reciprocal spaces, like the anomalous Hall effect in Hermitian systems. However, because of the gain and decay, we can expect the correct description to be essentially non-adiabatic, representing a superposition of branches, and thus not controlled by the Berry curvature any more.

In this work, we study the dynamics of WPs centered at an exceptional point, taking the Voigt point as a particular example. We demonstrate that the quantum metric of the eigenstates in the vicinity of exceptional points plays a dominant role in the WP dynamics, leading to a non-vanishing polarization-dependent group velocity.

\section{MODEL}

\section{A. Quantum metric}

We begin with the definition of the quantum metric, allowing to calculate the distances between the quantum states $^{10}$. In general, such distance $d s$ between the states $\psi(\lambda)$ and $\psi(\lambda+\delta \lambda)$ is linked with their overlap:

$$
d s^{2}=g_{i j} d \lambda_{i} d \lambda_{j}=1-|\langle\psi(\boldsymbol{\lambda}) \mid \psi(\boldsymbol{\lambda}+\delta \boldsymbol{\lambda})\rangle|^{2}
$$

where the metric tensor $g_{i j}$ can be found as a real part of the QGT, $g_{i j}=\operatorname{Re} T_{i j}$ :

$$
T_{i j}=\left\langle\frac{\partial \psi}{\partial \lambda_{i}} \mid \frac{\partial \psi}{\partial \lambda_{j}}\right\rangle-\left\langle\psi \mid \frac{\partial \psi}{\partial \lambda_{i}}\right\rangle\left\langle\frac{\partial \psi}{\partial \lambda_{j}} \mid \psi\right\rangle
$$

The overlap integral between an arbitrary state $|\psi\rangle$ and another state $\left|\psi_{1}\right\rangle$ can therefore be written using the metric tensor $g_{i j}$ as

$$
I=1-\left(\int_{|\psi\rangle}^{\left|\psi_{1}\right\rangle} \sqrt{g_{i j} d \lambda_{i} d \lambda_{j}}\right)^{2}
$$

where the integral should be taken along a geodesic line. These integrals determine the behavior of the coefficients $c_{l}(\boldsymbol{\lambda})\left(\left|c_{l}\right|^{2}=I\right)$ which define the representation of an arbitrary initial state $\psi$ as a superposition of the branches $l$ of the eigenstates $\left|\psi_{l}(\boldsymbol{\lambda})\right\rangle$ used in the general solution of the Schrödinger equation

$$
|\psi(\boldsymbol{\lambda}, t)\rangle=\sum_{l} c_{l}(\boldsymbol{\lambda}) e^{-i \frac{E_{l}(\boldsymbol{\lambda})}{\hbar} t}\left|\psi_{l}(\boldsymbol{\lambda})\right\rangle
$$
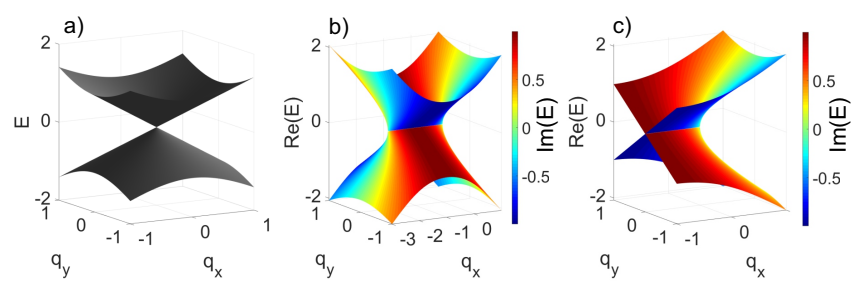

FIG. 1: a) Dispersion of a diabolical point (conical intersection). b) Transformation of a diabolical point into two exceptional points. c) Zoom on a single exceptional point at the origin. The false color shows the imaginary part of the energy.

\section{B. Diabolical and exceptional points}

We first consider a simple Hamiltonian with a diabolical point, which will serve as a reference:

$$
\hat{H}_{0}=\hbar c\left(\begin{array}{cc}
0 & q e^{-i \varphi} \\
q e^{i \varphi} & 0
\end{array}\right)=\hbar c \boldsymbol{q} \cdot \boldsymbol{\sigma}
$$

Here, $\boldsymbol{q}$ is a wave vector in polar coordinates $(\varphi$ is its polar angle), $c$ is the celerity, and $\boldsymbol{\sigma}$ is a vector of Pauli matrices. This 2D massless Dirac Hamiltonian, written for light on the circular basis, actually describes the oldest known diabolical point, dating back to Hamilton ${ }^{40}$ and Lloyd $^{41}$. The most striking manifestation of the diabolical point is the conical refraction, where a Gaussian WP is transformed into a ring. This is illustrated by Fig. 1(a) showing the conical intersection at the diabolical point. In microcavities, such diabolical points can arise from the combination of TE-TM splitting and birefringence ${ }^{11,42}$.

The dispersion is strongly modified in the presence of non-Hermitian terms in the Hamiltonian. Such terms can split a single diabolical point into two exceptional points (Fig. 1(b)). If these points are sufficiently far in the parameter space, it becomes possible to study each of them separately (Fig. 1(c)). We therefore consider a simplest Hamiltonian with an exceptional point in the center of the parameter space (reciprocal space), with $\hbar=1$ :

$$
\hat{H}_{1}=\left(\begin{array}{cc}
0 & \alpha q e^{-i \varphi}+a \\
\alpha q e^{i \varphi}+a & 0
\end{array}\right)+i\left(\begin{array}{cc}
0 & -i a \\
i a & 0
\end{array}\right)
$$

The first part of the Hamiltonian describes a real $q$ dependent Rashba-type ${ }^{42}$ effective field $\alpha q$ and a constant contribution $a$ along $x$. The second part is a nonHermitian imaginary part which is proportional to $\sigma_{y}$ and can be described as an imaginary effective field pointing along $y$. Its magnitude $a$ exactly equal to the constant real field along $x$. This configuration corresponds to the combination of TE-TM field, birefringence, and linear dichroism (polarization-dependent absorption) in the microcavities ${ }^{39}$. We note that the overall tilt of the Dirac cones at the crossing points ${ }^{11,42}$ does not affect the metric tensor, since it corresponds to a spin-independent 
part of the Hamiltonian. With the possible permutation of the Pauli matrices, the Hamiltonian (6) is equivalent to the Hamiltonians considered in other works ${ }^{6}$, and, in particular, to the gain-loss Hamiltonian ${ }^{24}$, and is in this sense completely general: up to the choice of the parameters and of the physical meaning of the eigenstates, it describes an arbitrary exceptional point in an arbitrary 2D parameter space.

The energy dispersion of this Hamiltonian is indeed very different from the case of the Dirac Hamiltonian: for small $q, E(q)= \pm \sqrt{2 a \alpha q} e^{-i \varphi / 2}$ (the so-called squareroot topology). The real part of the energy is shown in Fig. $1(\mathrm{~b}, \mathrm{c})$ as the $z$ coordinate. It determines the group velocity, which diverges as $1 / \sqrt{q}$. Moreover, the group velocity is, in general, not aligned with the wave vector. We note that the imaginary part of the energy (shown in Fig. 1(b,c) with false color) is complementary to the real part: $\operatorname{Re} E \sim \pm \sqrt{q} \cos \varphi / 2, \operatorname{Im} E \sim \pm \sqrt{q} \sin \varphi / 2$. It determines the decay or the growth of the corresponding states. The imaginary part is zero only along a single line in the parameter space (given by $\varphi=0$ ), ending at the exceptional point. For all other points, the states are either growing or decaying, which affects the behavior of WPs in dynamical experiments, as we will see below.

\section{RESULTS AND DISCUSSION}

\section{A. Circular-polarized WP}

Contrary to a diabolical point, associated with two degenerate eigenstates (which allows any linear combination of them to be a solution), an exceptional point corresponds to a single eigenstate. For the Hamiltonian (6) at $q=0$, this eigenstate is $\left|\psi_{0}\right\rangle=(1,0)^{T}$ : a circularlypolarized mode, typical for the Voigt points in optics. This can be generalized to exceptional points of any order, described by a Hamiltonian, that can be represented as a superposition of real and imaginary spin operators (that determine the complex effective field for spin $n$ ), because such superposition corresponds to the definition of the non-Hermitian ladder operator: $\hat{H} \sim \hat{S}_{x}+i \hat{S}_{y}=\hat{S}^{+}$, whose single eigenstate (with zero eigenvalue) is one of the eigenstates of $\hat{S}_{z}$.

We begin by considering a WP corresponding to the polarization of this eigenstate. It shows a finite wavevector distribution $\sigma_{q}$ (linked with the finite size of the exciting beam $\sigma_{r}=2 \pi / \sigma_{q}$ ) centered on the exceptional point.

A WP dynamics depends on its projections on the eigenstates of the two branches. The most convenient tool to describe this projection in this multi-level system is the Eq. (3) with the metric tensor. Let us start by calculating the radial component of the metric $g_{q q}$. For small $q$, the eigenstates of (6) behave as
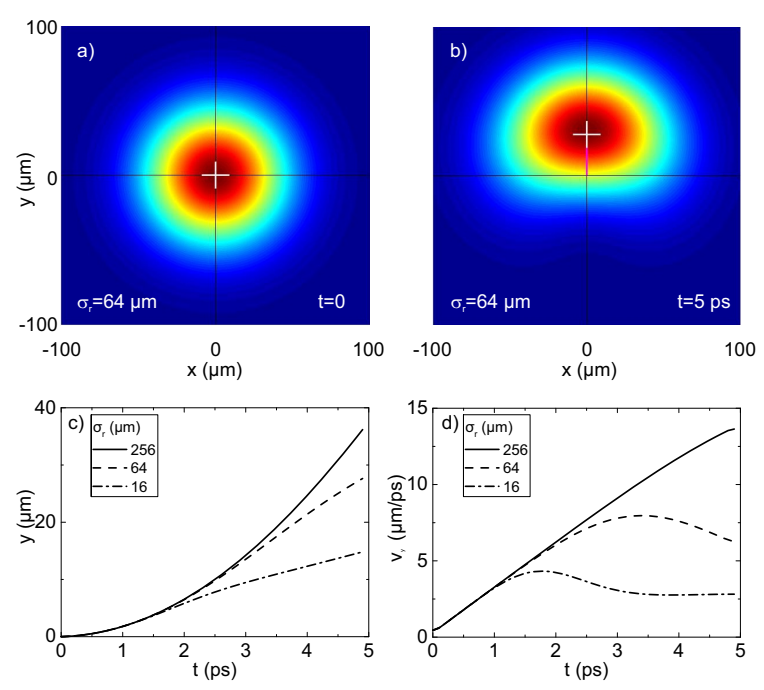

FIG. 2: Circular-polarized WP at an exceptional point. (a,b) The probability density distribution $|\psi(x, y, t)|^{2}$ for a circularpolarized initial wavepacket of $\sigma_{r}=64 \mu \mathrm{m}$ at $t=0$ (a) and $t=5 \mathrm{ps}$ (b). The center of mass is shown with a white cross and its trajectory is shown with a magenta line. c) The center of mass position as a function of time. d) The center of mass velocity as a function of time, demonstrating constant acceleration. Solid, dashed, and dash-dotted lines correspond to different WP sizes.

$\left|\psi_{q}\right\rangle=\left(1-\alpha q / 4 a, e^{i \varphi / 2} \sqrt{\alpha q / 2 a}\right)^{T}$, which allows to find:

$$
g_{q q} \approx \frac{\alpha^{2}}{16 a^{2}}+\frac{\alpha}{8 a q}
$$

using Eq. (2). This $q^{-1}$ divergence of the metric is a general result, valid for any second-order exceptional point ${ }^{9}$. We note that the divergence can be different for higherorder exceptional points, which will be discussed in a separate work. We also note that the other component of the metric tensor $g_{\varphi \varphi}$, whose divergence determines the dynamics of a WP center on a diabolical point, is now linear in $q: g_{\varphi \varphi} \approx \alpha q / 8 a$. Since the metric $g_{q q}$ does not depend on $\varphi$, the equation for the overlap integral between the states $\left|\psi_{0}\right\rangle$ and $\left|\psi_{q}\right\rangle$ can be explicitly written as:

$$
I_{\text {circ }}=1-\left(\int_{0}^{q} \sqrt{g_{q q}(d q)^{2}}\right)^{2} \neq f(\varphi)
$$

Since there is no dependence on $\varphi$, the WP is initially symmetrically distributed around the exceptional point on both branches. However, each individual state starts to grow or decline according to the imaginary part of its energy $\operatorname{Im} E$. The highest growth and decay rates are observed along the line $\varphi=\pi$, where $\sin \varphi / 2=1$. The group velocity for all points along this line is perpendicular to the wave vector. It behaves as $v_{y} \sim q_{x}^{-1 / 2}$ (see the Appendix for details). At the same time, the growth and 
decay rates of the bands behave as $\Gamma \sim \pm q_{x}^{1 / 2}$. The growing and decaying parts of the WP $\left(n_{+}\right.$and $\left.n_{-}\right)$belong to different branches propagating in opposite directions along $y$. The average WP velocity for sufficiently small times and WP size is therefore given by

$$
\left\langle v_{y}\right\rangle=\left(n_{+}-n_{-}\right) v_{y} \approx 2 v_{y} \Gamma t \approx 2 \sqrt{2} \alpha a t
$$

which does not depend on the wave vector $q_{x}$, because the dependencies of $v_{y}$ and $\Gamma$ are inverse and compensate each other. We can therefore expect a finite-size WP centered at the eigenstate at the exceptional point to exhibit a constant acceleration in the vertical direction, because all of its components exhibit equal acceleration. This acceleration is proportional to the celerity $\alpha$ and the dichroism $a$.

This is confirmed by direct numerical simulations. We solve the time-dependent spinor Schrödinger equation with the Hamiltonian $\hat{H}_{1}$ defined by Eq. (6) and extract the center of mass position as a function of time for different initial size of the WP, taking the parameters of the perovskite microcavity exhibiting a large birefringence ${ }^{43}$. The results of the simulations are shown in Fig. 2. We present two snapshots of the particle density $|\psi(x, y, t)|^{2}$ taken at the initial (panel (a), $t=0$ ) and final (panel (b), $t=5 \mathrm{ps}$ ) moments of time for a wave packet of $\sigma_{r}=64 \mu \mathrm{m}$. The center of mass of the wave packet is shown with a white cross, and its trajectory is shown with a magenta line in panel (b). The displacement of the center of mass is clearly visible. The wave packet is also distorted; this distortion is stronger if its size $\sigma_{r}$ is smaller. For a larger wave packet of $\sigma_{r}=256 \mu \mathrm{m}$ this distortion is not visible by a naked eye for the same time interval. Panel (c) shows the evolution of the $y(t)$ coordinate of the center of mass as a function of time, which is clearly parabolic, and panel (d) shows the center of mass velocity $v_{y}(t)$, which grows linearly, as expected. The acceleration corresponds well to the analytical solution (9). Changing the size of the WP in the real space $\sigma_{r}$ (as shown by the line style in Fig. 2(c,d)) also leads to a very interesting and counter-intuitive behavior. Indeed, the linear increase of the velocity occurs only while the populations of the two branches $n_{+}$and $n_{-}$are comparable. The duration of this regime is determined by the maximal gain/loss ratio available within the WP size in the reciprocal space $\sigma_{q}$. For high $\sigma_{q}$, the regime of linear increase is lost more rapidly. While the wave vector of the center of mass $q_{0}$ of the WP is at this moment higher than for a smaller $\sigma_{q}$, the corresponding group velocity is lower, because $v_{g} \sim 1 / \sqrt{q_{0}}$. So, a WP which is larger in reciprocal space (dash-dotted line) exhibits a smaller final velocity and a shorter acceleration period (and, finally, a smaller total displacement for the same amount of time).
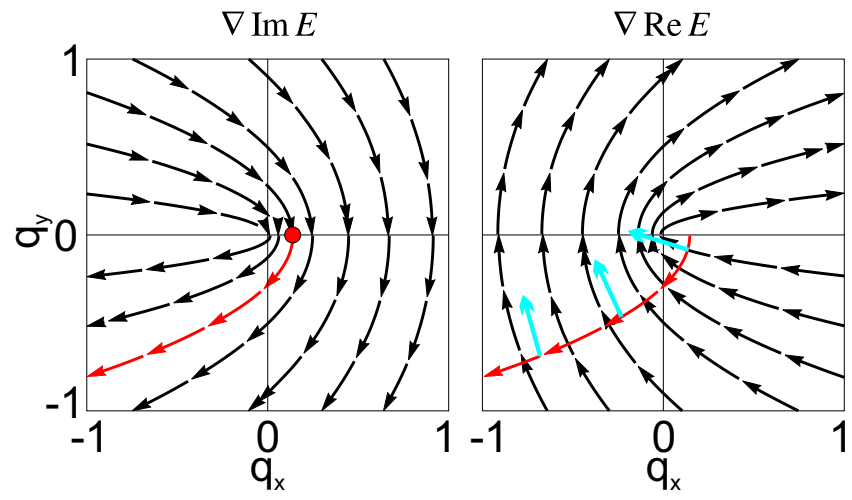

FIG. 3: a) The gradient of the imaginary part of the energy $\nabla \operatorname{Im} E$ for one dispersion branch. The arrows show how the center of mass of the WP moves in the reciprocal space due to the amplification of its components. The linear-polarized WP projection of the branch is marked with a red circle. b) The group velocity map $\nabla \operatorname{Re} E$ with the trajectory of the center of mass in red, and the corresponding group velocities highlighted in blue.

\section{B. Linear-polarized WP}

As shown above, the quantum metric tensor $g_{q q}$ does not depend on $\varphi$, and thus the circular-polarized WP centered on the exceptional point exhibited exactly the same overlap with both branches at any $q$. Because of this, we had $n_{+}=n_{-}$at $t=0$, and the initial WP velocity was zero. The situation is different if the polarization of the WP does not correspond to the eigenstate of the exceptional point. As said above, the metric is divergent, which means that the eigenstates change very rapidly with $q$. Because of this, even an infinitely small (in reciprocal space) linear-polarized WP exhibits a different overlap with the two branches. Moreover, its center of mass on each branch is not centered at $q=0$ any more. Indeed, using Eq. (3), the overlap of the eigenstates with the H-polarized excitation spinor $\left|\psi_{0}\right\rangle=(1 / \sqrt{2}, 1 / \sqrt{2})^{T}$ can be found as:

$$
I_{l i n} \approx \frac{1}{2}+\int_{|\psi(0)\rangle}^{|\psi(q)\rangle} \sqrt{g_{q q}} d q \approx \frac{1}{2}+\sqrt{\frac{\alpha q}{2 a}}
$$

where we have used trigonometric identities to express the integral as a function of the metric at small $q>0$ $\left(I_{\text {lin }}=1 / 2\right.$ for $\left.q \leq 0\right)$. The fast growth of the overlap leads to a non-zero effective center-of-mass wave vector $q_{0}$ for a Gaussian WP of any size, behaving as $q_{0} \sim \sigma_{q}^{3 / 2} \sqrt{\alpha / 2 a}$ for small $\sigma_{q}$ (large WPs in real space, small in reciprocal space). The position of this center of mass is determined by the polarization of the WP. This starting point is shown in Fig. 3 with a red circle. The evolution of the WP over time in the reciprocal space is dictated by the imaginary part of the energy: the center of mass $\boldsymbol{q}_{\mathbf{0}}$ shifts in the direction of the gradient $\nabla \operatorname{Im} E$, 
as shown in Fig. 3(a) for a WP with initial $\mathrm{H}$ polarization (red arrows). The ratio of the components of the gradient of the imaginary part of the energy $\left(\eta_{x}, \eta_{y}\right)=\nabla \operatorname{Im} E$ reads simply $\eta_{y} / \eta_{x}=\left(-q_{x}+\sqrt{q_{x}^{2}+q_{y}^{2}}\right) / q_{y}$. For an initial wave vector $q_{0}$, this gives a parabolic trajectory $q_{x}=-\left(q_{y}^{2}-q_{0}^{2}\right) /\left(2 q_{0}\right)$. For sufficiently large $q_{x}$, one finds that $\left|q_{y}\right| \approx \sqrt{2 q_{0}\left|q_{x}\right|}$. The associated group velocity changes over time (blue arrows in Fig. 3(b)), according to the position of the WP in the reciprocal space. The velocity of the very first moments in time corresponds to the high-energy terms of the Hamiltonian (celerity $\alpha$ ), which are determined by its $q$-dependent Hermitian part. Then, the velocity drops to the value determined by the ratio of the populations of the two bands $n_{+}$and $n_{-}$, and the respective positions of the WP projections in the two bands. The $v_{x}$ projection of the group velocity for very small WPs (and $q_{y} \ll q_{x}$ ) is $v_{x} \approx 3 \sqrt{\alpha a} q_{y}^{2} / 8 q_{x}^{2}$ (see the Appendix). To obtain the time dependence of the group velocity, we need to use the center of mass wave vector $q_{x}(t)$, whose calculation again involves the quantum metric via the overlap integral $I_{\text {lin }}$. For small $t, q_{x}(t) \approx \alpha a q_{0}^{2} t^{2} / \sqrt{2 \pi}$, and finally, combining all coefficients, the group velocity tends to a constant nonvanishing value

$$
\left\langle v_{x}\right\rangle \approx 0.38 \alpha
$$

which does not depend on the WP size. Moreover, it only depends on the celerity $\alpha$ of the Hermitian part of the Hamiltonian, and not on the non-Hermitian dichroism parameter $a$.

All this is indeed confirmed by numerical simulations. We illustrate the motion of the linear-polarized wave packet in real space by showing its initial and final density distributions in Fig. 4(a,b). The center of the wave packet is shown with a white cross, and its trajectory appears as a magenta line. A strong displacement along the vertical direction, which was already present for a circular-polarized wavepacket, is now accompanied by a smaller lateral displacement. Figure 4(c) shows all possible trajectories for WPs of different polarizations. They all demonstrate a constant acceleration along $y$, as for a circular WP. An additional polarization dependent constant velocity contribution appears. For an H-polarized WP (black circles in panel (c)), it is directed along $x$ and therefore can be studied independently from the other contribution $v_{y}$. Fig. $4(\mathrm{~d})$ shows that the velocity $v_{x}$ of an H-polarized WP quickly drops from the value given by the Hermitian part of the Hamiltonian $\left(v_{x}=\alpha\right)$ at $t=0$ down to the constant value predicted by (11) and indeed independent of the dichroism $a$ (dashed line). This finite constant velocity differs drastically from the behavoir of a gapped Dirac Hamiltonian, where the radial metric decays as $g_{q q} \sim-q^{2}$ and therefore does not diverge. Because of this, the associated group velocity tends to zero in the limit of a WP infinitely large in real space $v_{\text {Dirac }} \sim 1 / \sigma_{r}^{2} \rightarrow 0$. Contrary to a diabolical point, associated with a localized Berry curvature (delta
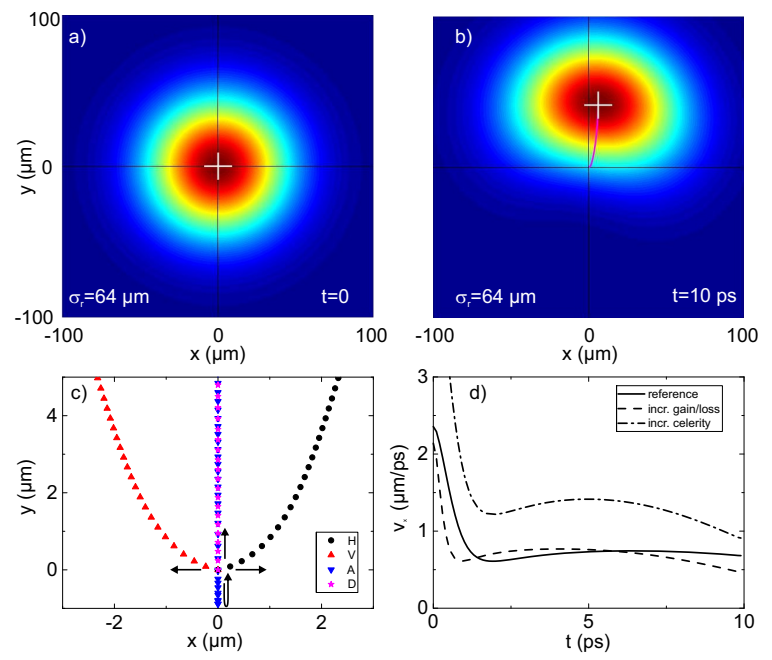

FIG. 4: Linear-polarized WP. (a,b) The probability density distribution $|\psi(x, y, t)|^{2}$ for an initial wavepacket of $\sigma_{r}=$ $64 \mu \mathrm{m}$ at $t=0$ (a) and $t=10 \mathrm{ps}$ (b). The center of mass is shown with a white cross and its trajectory is shown with a magenta line. c) Polarization-dependent center of mass trajectories. d) The $x$-projection of the H-polarized WP velocity over time (solid curve - same parameters as in (a); dashed curve - increased $a$ (gain/losses); dash-dotted curve increased celerity $\alpha$ ).

function), an exceptional point exhibits distributed Berry curvature $^{7,8}$. However, dynamical effects associated with this Berry curvature, such as the anomalous Hall effect, are practically unobservable, because the divergent group velocity dominates all possible corrections.

The effects we have described can be measured optically in microcavities with exceptional points ${ }^{39}$, in atomic vapors $^{28}$, or in dichroic birefringent crystals $^{35}$, but the theory we developed applies to all 2nd-order exceptional points. For example, such dynamics can be studied in photonic crystals, so far as they exhibit well-defined exceptional points in the Brillouin zone. The main requirement is that the two exceptional points forming a single couple should be well-resolved. For example, in Ref. ${ }^{39}$ the distance between such points was approximately $8 \mu \mathrm{m}^{-1}$, which means that the wavepacket size in real space has to be much larger than $2 \mu \mathrm{m}$. This is not a stringent condition at all: for example, the $30 \mu \mathrm{m}$ wavepackets, whose behavior was studied in ${ }^{11}$, clearly satisfy this condition. Similar effects can be expected for higher-order exceptional points, but we leave a detailed study for the future works. On the other hand, the behavior of WPs around Weyl exceptional rings is probably different ${ }^{44}$. We note that contrary to the anomalous Hall effect, recently observed in microcavities ${ }^{11}$, the WP displacement in the present configuration does not saturate and continues to grow with time or propagation distance.

These results demonstrate that the quantum metric plays a particularly important role in vicinity of the exceptional points, determining the dynamical behavior of 
WPs. The crucial feature is that the radial component of the quantum metric diverges. This, together with the divergent group velocity, leads to a non-vanishing polarization-dependent velocity for any finite-size WP, centered at the exceptional point. Our studies are important for future research and applications in nonHermitian photonics.

\section{Acknowledgments}

We acknowledge the support of the projects EU "QUANTOPOL" (846353), "Quantum Fluids of Light" (ANR-16-CE30-0021), of the ANR Labex GaNEXT (ANR-11-LABX-0014), and of the ANR program "Investissements d'Avenir" through the IDEX-ISITE initiative 16-IDEX-0001 (CAP 20-25).

\section{APPENDIX A: CIRCULAR WAVEPACKET}

To find the group velocity, one has first to determine the dispersion, then to take its derivative, and then to fix the wave vector, at which the value of the group velocity is needed. We therefore keep both components of the wave vector as variables at first, and only in the end of the calculation set $q_{y}=0$. We write the series expansion of the real part of the energy for small $q$ along the line $\varphi=$ $\pi$, where we use the identity $\cos \varphi / 2=\sqrt{(1+\cos \varphi) / 2}$ :

$$
E(\boldsymbol{q}) \approx \sqrt{2 \alpha a}\left(q_{x}^{2}+q_{y}^{2}\right)^{1 / 4} \sqrt{\frac{1+\frac{q_{x}}{\sqrt{q_{x}^{2}+q_{y}^{2}}}}{2}} \approx \sqrt{\alpha a} \frac{q_{y}}{\left|q_{x}\right|^{1 / 2}}
$$

Here, we have first used that $q$ is small, and then that $q_{y} \ll q_{x}$, and also that $\left|q_{x}\right|=-q_{x}$ for $q_{x}<0$. The group velocity in the vertical direction is given by $v_{y}=\partial E / \partial q_{y}$, which gives the expression

$$
v_{y} \approx \frac{\sqrt{\alpha a}}{\left|q_{x}\right|^{1 / 2}}
$$

used in the main text.

\section{APPENDIX B: LINEARLY-POLARIZED WAVEPACKET}

The effective center of mass wave vector for one of the branches at $t=0$ is determined as:

$$
q_{0}=\int q \times I_{l i n} \times\left|\psi_{0}(q)\right|^{2} d q
$$

where $\left|\psi_{0}(q)\right|^{2}$ is the excitation wavefunction (Gaussian WP with $\sigma_{q}$ width), $I_{\text {lin }}$ is the overlap integral with the chosen branch (given in the main text). The integral has to be divided into two parts, $q \leq 0$ and $q>0$. The contributions with $I_{l i n}=1 / 2$ compensate each other,
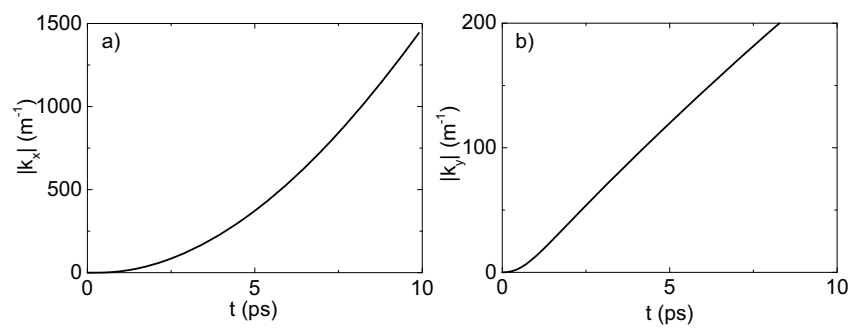

FIG. A1: The center of mass wave vector as a function of time, obtained from numerical simulations for a linearly-polarized wave packet: a) $k_{x}(t)$ (parabolic growth), b) $k_{y}(t)$ (linear growth).

while the remaining term for $q>0$ gives the dependence $q_{0} \sim \sigma_{q}^{3 / 2}$ indicated in the main text.

To find the expression for the group velocity projection on the $x$ axis, we use the expression for the real part of the energy once again

$$
E(\boldsymbol{q}) \approx \sqrt{2 \alpha a}\left(q_{x}^{2}+q_{y}^{2}\right)^{1 / 4} \sqrt{\frac{1+\frac{q_{x}}{\sqrt{q_{x}^{2}+q_{y}^{2}}}}{2}}
$$

which we derive over $q_{y}$ and then apply series expansion over $q_{y}$, taking care of $q_{x}<0$. This gives the expression for $v_{x} \approx 3 \sqrt{\alpha a} q_{y}^{2} / 8 q_{x}^{2}$ given in the main text.

For the calculation of $q_{x}(t)$ (the $x$ projection of the effective center of mass coordinate), we take into account that the difference of the population of the branches is controlled by the overlap integral, which behaves as $I_{\text {lin }} \approx 1 / 2+\sqrt{\alpha q_{x} / 2 a}$ and by the imaginary part $\Gamma t \sim$ $\sqrt{q_{x}} t$. The position of the center of mass on a given branch is therefore given by

$$
\left\langle q_{1}\right\rangle \sim \int q(1+q t)\left|\psi_{0}(q)\right|^{2} d q \sim \sigma_{q}^{2} t
$$

while the population of this branch is given by

$$
I_{1} \sim \int(1+q t)\left|\psi_{0}(q)\right|^{2} d q \sim \sigma_{q} t
$$

while the sum of the two branches $I_{1}+I_{2}$ remains approximately constant (one branch grows, the other decays). We therefore find that

$$
q_{x}(t) \sim \sigma_{q}^{3} t^{2}
$$

where $\sigma_{q}^{3} \sim q_{0}^{2}$ (the initial wave vector of a WP within one of the branches). This allows to find $v_{x}$ using $q_{x}(t)$ and $q_{y}=\sqrt{2 q_{0} q_{x}}$.

The results concerning the dynamics of a linearpolarized wavepacket, provided in the main text, are strongly based on its center of mass position in the reciprocal space. As an additional confirmation of our theoretical considerations, we provide the numerical results on the center of mass wave vector in this Appendix. Figure A1 shows the two projections of the center of mass 
wavevector as a function of time. Panel (a) clearly exhibits a parabolic growth (which is valid both for circular and linear wavepackets), whereas panel (b) demonstrates a linear dependence on time. This is due to the fact that the center of mass wave vector follows the trajectories of maximal gradient of the imaginary part of the energy shown in Fig. 3 of the main text, which represent a family of parabolic curves, and therefore $k_{y} \sim \sqrt{k_{x}} \sim t$. The parameters were the same as in Fig. 4 of the main text.
${ }^{1}$ N. Moiseyev, Non-Hermitian quantum mechanics (Cambridge University Press (Cambridge, UK), 2011).

${ }^{2}$ Ş. Özdemir, S. Rotter, F. Nori, and L. Yang, Nature materials 18, 783 (2019).

${ }^{3}$ V. V. Konotop, J. Yang, and D. A. Zezyulin, Rev. Mod. Phys. 88, 035002 (2016), URL https://link.aps.org/ doi/10.1103/RevModPhys.88.035002.

${ }^{4}$ R. El-Ganainy, K. G. Makris, M. Khajavikhan, Z. H. Musslimani, S. Rotter, and D. N. Christodoulides, Nature Physics 14, 11 (2018).

${ }^{5}$ H. Shen, B. Zhen, and L. Fu, Phys. Rev. Lett. 120, 146402 (2018), URL https://link.aps.org/doi/ 10.1103/PhysRevLett.120.146402.

${ }^{6}$ D. Leykam, K. Y. Bliokh, C. Huang, Y. D. Chong, and F. Nori, Phys. Rev. Lett. 118, 040401 (2017), URL https : //link.aps.org/doi/10.1103/PhysRevLett.118.040401.

7 X.-D. Cui and Y. Zheng, Phys. Rev. A 86, 064104 (2012), URL https://link.aps.org/doi/10.1103/PhysRevA. 86.064104 .

8 Q. Zhang and B. Wu, Phys. Rev. A 99, 032121 (2019), URL https://link.aps.org/doi/10.1103/PhysRevA. 99.032121.

9 D. C. Brody and E. M. Graefe, Entropy 15, 3361 (2013), ISSN 10994300, 1307.4017.

10 J. Provost and G. Vallee, Communications in Mathematical Physics 76, 289 (1980).

11 A. Gianfrate, O. Bleu, L. Dominici, V. Ardizzone, M. De Giorgi, D. Ballarini, G. Lerario, K. West, L. Pfeiffer, D. Solnyshkov, et al., Nature 578, 381 (2020).

12 M. Yu, P. Yang, M. Gong, Q. Cao, Q. Lu, H. Liu, S. Zhang, M. B. Plenio, F. Jelezko, T. Ozawa, et al., National Science Review 7, 254 (2019), ISSN 2095-5138, https://academic.oup.com/nsr/articlepdf/7/2/254/32921980/nwz193.pdf, URL https://doi. org/10.1093/nsr/nwz193.

13 P. Zanardi, P. Giorda, and M. Cozzini, Phys. Rev. Lett. 99, 100603 (2007), URL https://link.aps.org/doi/10. 1103/PhysRevLett.99.100603.

14 Y. Gao, S. A. Yang, and Q. Niu, Phys. Rev. Lett. 112, 166601 (2014), URL https://link.aps.org/doi/ 10.1103/PhysRevLett.112.166601.

15 F. Piéchon, A. Raoux, J.-N. Fuchs, and G. Montambaux, Phys. Rev. B 94, 134423 (2016), URL https ://link.aps . org/doi/10.1103/PhysRevB.94.134423.

16 A. Srivastava and A. Imamoglu, Phys. Rev. Lett. 115, 166802 (2015), URL http://link.aps.org/doi/10.1103/ PhysRevLett.115.166802.

17 S. Peotta and P. Törmä, Nature communications 6, 8944 (2015).

18 L. Liang, S. Peotta, A. Harju, and P. Törmä, Phys. Rev. B 96, 064511 (2017), URL https://link.aps.org/doi/10. 1103/PhysRevB.96.064511.

19 O. Bleu, G. Malpuech, Y. Gao, and D. D. Solnyshkov, Phys. Rev. Lett. 121, 020401 (2018), URL https://link.
aps.org/doi/10.1103/PhysRevLett.121.020401.

20 Q. Zhong, M. Khajavikhan, D. N. Christodoulides, and R. El-Ganainy, Nature communications 9, 1 (2018).

21 M. Berry and R. Uzdin, Journal of Physics A: Mathematical and Theoretical 44, 435303 (2011).

22 M. Berry, Journal of Optics 13, 115701 (2011).

23 T. J. Milburn, J. Doppler, C. A. Holmes, S. Portolan, S. Rotter, and P. Rabl, Phys. Rev. A 92, 052124 (2015), URL https://link.aps.org/doi/10. 1103/PhysRevA.92.052124.

24 J. Doppler, A. A. Mailybaev, J. Böhm, U. Kuhl, A. Girschik, F. Libisch, T. J. Milburn, P. Rabl, N. Moiseyev, and S. Rotter, Nature 537, 76 (2016).

25 X. L. Zhang, S. Wang, B. Hou, and C. T. Chan, Physical Review X 8, 21066 (2018), ISSN 21603308, 1804.09145, URL https://doi.org/10.1103/PhysRevX.8.021066.

${ }^{26}$ M. V. Berry and P. Shukla, Journal of Physics A: Mathematical and Theoretical 53, 275202 (2020), URL https: //doi.org/10.1088\%2F1751-8121\%2Fab91d6.

27 C. Hahn, Y. Choi, J. W. Yoon, S. H. Song, C. H. Oh, and P. Berini, Nature Communications 7, 1 (2016), ISSN 20411723

${ }^{28}$ Z. Zhang, Y. Zhang, J. Sheng, L. Yang, M.-A. Miri, D. N. Christodoulides, B. He, Y. Zhang, and M. Xiao, Phys. Rev. Lett. 117, 123601 (2016), URL https://link.aps.org/ doi/10.1103/PhysRevLett.117.123601.

29 J. Wiersig, Physical Review A 93, 1 (2016), ISSN 24699934.

30 M. Naghiloo, M. Abbasi, Y. N. Joglekar, and K. W. Murch, Nature Physics 15, 1232 (2019), ISSN 17452481, 1901.07968, URL http://dx.doi.org/10. 1038/s41567-019-0652-z.

31 A. Kavokin, J. J. Baumberg, G. Malpuech, and F. P. Laussy, Microcavities (Oxford University Press, 2011).

32 T. Gao, E. Estrecho, K. Bliokh, T. Liew, M. Fraser, S. Brodbeck, M. Kamp, C. Schneider, S. Höfling, Y. Yamamoto, et al., Nature 526, 554 (2015).

33 T. Gao, G. Li, E. Estrecho, T. C. H. Liew, D. ComberTodd, A. Nalitov, M. Steger, K. West, L. Pfeiffer, D. W. Snoke, et al., Phys. Rev. Lett. 120, 065301 (2018), URL https://link.aps.org/doi/10. 1103/PhysRevLett.120.065301.

${ }^{34}$ W. Voigt, Philosophical Magazine Series 4, 90 (1902).

35 M. V. Berry and M. R. Dennis, Proc. R. Soc. Lond. A 459, 1261 (2003).

${ }^{36}$ L. D. Landau and E. M. Lifshitz, Electrodynamics of Continuous Media (Butterworth-Heinemann, 1984).

37 C. Sturm, V. Zviagin, and M. Grundmann, Phys. Rev. Materials 4, 055203 (2020), URL https://link .aps.org/ doi/10.1103/PhysRevMaterials.4.055203.

38 S. Richter, T. Michalsky, C. Sturm, B. Rosenow, M. Grundmann, and R. Schmidt-Grund, Phys. Rev. A 95, 023836 (2017), URL https://link.aps.org/doi/10. 1103/PhysRevA.95.023836. 
39 S. Richter, H.-G. Zirnstein, J. Zúñiga Pérez, E. Krüger, C. Deparis, L. Trefflich, C. Sturm, B. Rosenow, M. Grundmann, and R. Schmidt-Grund, Phys. Rev. Lett. 123, 227401 (2019), URL https://link.aps.org/doi/10. 1103/PhysRevLett.123.227401.

${ }^{40}$ W. R. Hamilton, Trans. Royal Irish Acad. 17, 1 (1837).

41 H. Lloyd, Trans. Roayl Irish Acad. 17, 145 (1837).

42 H. Terças, H. Flayac, D. D. Solnyshkov, and G. Malpuech, Phys. Rev. Lett. 112, 066402 (2014), URL https://link.
aps.org/doi/10.1103/PhysRevLett.112.066402.

43 A. Fieramosca, L. Polimeno, G. Lerario, L. De Marco, M. De Giorgi, D. Ballarini, L. Dominici, V. Ardizzone, M. Pugliese, V. Maiorano, et al., arXiv:1912.09684 (2019). 44 Y. Xu, S.-T. Wang, and L.-M. Duan, Phys. Rev. Lett. 118, 045701 (2017), URL https://link.aps.org/doi/ 10.1103/PhysRevLett.118.045701. 